\title{
Constrained discrete model predictive control of a greenhouse system temperature
}

\author{
Hafsa Hamidane ${ }^{1}$, Samira El Faiz ${ }^{2}$, Mohammed Guerbaoui ${ }^{3}$, Abdelali Ed-Dahhak ${ }^{4}$, Abdeslam \\ Lachhab $^{5}$, Benachir Bouchikhi ${ }^{6}$ \\ ${ }^{1,3,4,5}$ Modelling, Materials and Control of Systems Team, High School of Technology, Moulay Ismaïl University, Meknes, \\ Morocco \\ ${ }^{2}$ Energy and Sustainable Development Research Team, High School of Technology, Ibn Zohr University, Guelmim, \\ Morocco \\ ${ }^{6}$ Sensor Electronic and Instrumentation Team, Faculty of Sciences, Moulay Ismaïl University, Meknes, Morocco
}

\begin{tabular}{|c|c|}
\hline Article Info & ABSTRACT \\
\hline Article history: & In this paper, a constrained discete model predictive control (CDMPC) strategy for \\
\hline Received Mar 9, 2020 & a greenhouse inside temperature is presented. To describe the dynamics of our sys- \\
\hline Revised Jul 27, 2020 & tem's inside temperature, an experimental greenhouse prototype is engaged. For the \\
\hline Accepted Aug 7, 2020 & $\begin{array}{l}\text { greenhouse temperature dynamics is identified using the subspace system identifica- } \\
\text { tion (N4sid) algorithm. The obtained model is used in order to develop the CDMPC }\end{array}$ \\
\hline Keywords: & starategy which role is to select the best control moves based on an optimization pro- \\
\hline Constraints & cedure under the constraints on the control notion. For efficient evaluation of the pro- \\
\hline Greenhouse & for algorithm and blocks implementation. The simulation results confirm the accuracy \\
\hline Linear system & of the controller that garantees both the control and the reference tracking objectives. \\
\hline
\end{tabular}

Model predictive control

Temperature control

Optimization

Yalmip

This is an open access article under the CC BY-SA license.

\section{Corresponding Author:}

Hafsa Hamidane

Modelling, Materials and Control of Systems

Team, High School of Technology, Moulay Ismail University

B.P. 11201, Zitoune, 50003, Meknes, Morocco

Email: Hamidanehafsa@gmail.com

\section{INTRODUCTION}

Nowadays agricultural green houses industry is considered as one of the most important and growing segment of all agri-food domains. In fact seeking new varieties, sustainable, high-performing and affordable production methods to create powerful yields and higher quality of the plants, and to reduce the industry's impact on the environment have always had a stronghly held value in agricultural economics and innovation of all time. The control of the climatic environment indooor greenhouses has gained considerable attention in the past few years $[1,2]$. The main reasons for this increasing interest are related to different factors one to be cited agronomic and financial ones.

As a matter of fact, a large number of methods regarding the control of the climatic conditions under greenhouses has been developed and elaborated, hence several teams in applied research have experienced this techniques to fathom and to enhance green houses control outstanding, among them: the fuzzy control [3, 4], predictive control [5, 6], in addition to neuronal networks control [7, 8], optimal control [9] and many other tecniques that have emerged in many litterature articles. In the control theory, model predictive control has 
emerged many research and development areas. Thanks to its advantages and roles, this control technique has been present in various industrial process automation [10-12], among of them the control of greenhouses's inside climate, one can refer to $[13,14]$ and reference therein.

Furthermore, Model predictive control (MPC) is a widely used method for a large variety of systems, its simplicity of use makes it applicable for single, multivariable, linear and nonlinear systems and where the notion of nonlinearities and constraints incorporation, such as limitations on the sign and amplitude of the states and controls; regarding the control law synthesis; is always involved. In this sense, designing controllers that maintain the system's performances regarding these constraints is a topic of continuing evolution, hence several are the MPC approaches that have been suggested and studied by researchers. For instance, we can refer to [15-21] and many others as well. The problem adressed in our framework, is related to regulation task of inside greenhouse temperature, among various modern control strategies, model based predictive control is choosen as a technique to overcome this problem.

The main purpose of the proposed control theory, is to calculate an objective and quadratic cost function over a finite horizon of the current state and control trajectory, while satisfying constraints on the control. To do so, an algorithm develloped using Yalmip optimization toolbox [22] in the form of an object oriented code is used simultaneously with an interpreted MATLAB function block that will hold this latest for simulation purposes under Simulink. The control law synthesis using a new toolbox as Yalmip together with Simulink models and blocks allow in one hand respecting the main CDMPC strategy and in another hand minimizing overhead and unneeded calculations by using an optimizer object, thing that was not treated before, regarding our inside climate parameter control case of study.

It's worth mentioning to point that the particular novelties of the present paper could be summarized as follows:

- Using an optimal method as MPC to automatically control inside climatic parameters for industrual greenhouse as a complex system.

- The utility of MPC as a perfect control approach that allows direct incorporation of constraints to an objective function.

- Providing the control activities using an optimizer with Yalmip optimization toolbox that incorporates an efficient technique which solves the problem as fast as possible.

The remaining of the paper is structured as follows, The second section steps through the greenhouse model identification and a reminder of CDMPC purposes and controller strategy, including optimization aims, parameters choice and constraints notions, in addition to the main principles of the algorithm used in our work. In the third section simulation results and discussion related to the CDMPC design strategy and synthesis will be provided. Conclusions and some of our future perspectives will be presented at the last section of this paper.

\section{ENGAGED MATERIALS AND METHODS}

\subsection{Description of the greenhouse system prototype}

As depicted in Figure 1 the experimental greenhouse engaged as support in this framework is a prototype installed at the Laboratory of Electronics, Automatics and Biotechnology (LEAB), Faculty of Sciences, Meknes, Morocco. This system is a polyethylene single wall construction, equipped with four sensors that provide indoor and outdoor measurements of temperature and relative humidity. More consicely, a LM35DZ and a HIH-4000-001 Honeywell sensors are installed to provide respectivelly the indoor/outdoor temperature and relative humidity measurements. Besides, several actuators are present as well; a heating system and a thermostatically variable speed fan; equipied the greenhouse to insure the appropriate climate for the system's inside parameters.

For control and data acquisition aims, the mentionned sensors and actuators are connected to a control and acquisition cards attached to a personal computer [23]. Firstly, the acquisition of the actuators different orders and data are ensured by an acquisition data card NI-PCI6024E from Advantech family. In this regard signals conditionning, protection and power cards dedicated to the sensors and the hole system protection, are also installed. Secondly, the control and supervision tasks; that manage the system and provide a historical database of both measured indoor and outdoor climate variables; are created respectively under MATLAB/Simulink and Labview as software programs. 


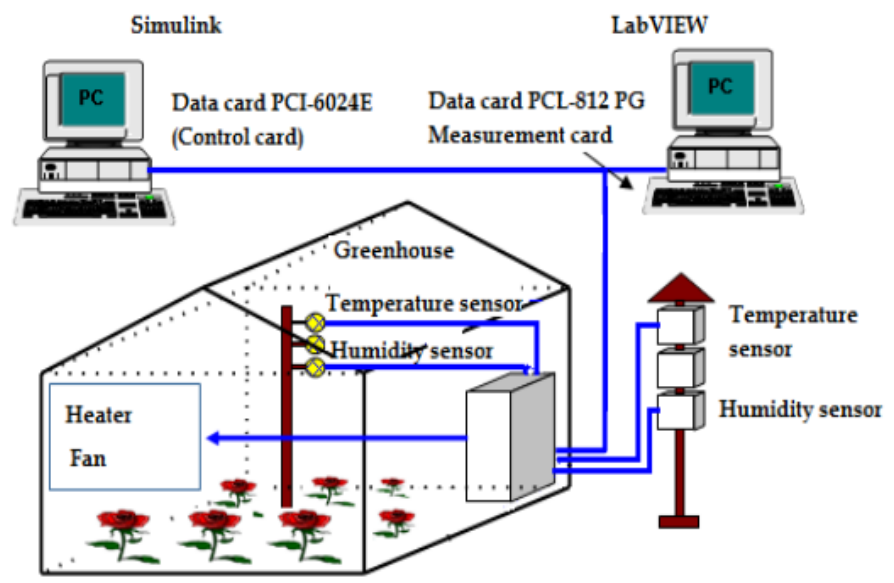

Figure 1. Experimental greenhouse system

\subsection{Mathematical model for controller design}

In the present section, a mathematical model of the inside temperature under greenhouse has been presented. Regarding this, a state space model describing; the greenhouse inside temperature dynamic response to the Fan as first actuator and then to the heater as second actuator; is proposed. The model used will enable us to modify the behavior of the plant to suit our needs in term of reference temperature tracking and control performances. In order to develop the controller synthesis and behavior, the plant model has to be obtained. For this aim, the system model is estimated using experimental collected data and the N4sid algorithm to identify the plant in discrete time state space model that describes the behavior of the inside temperature of the greenhouse.

For linear subspace identification, systems and models of the form (1), are generaly used.

$$
\left\{\begin{aligned}
x_{k+1} & =A x_{k}+B u_{k}+K w_{k} \\
y_{k} & =C x_{k}+D u_{k}+v_{k}
\end{aligned}\right.
$$

Where $x_{k}, u_{k}, y_{k}, w_{k}$ and $v_{k}$ are respectivelly the state, input, output, process and the output measurement noises vectors and $A, B, C, D, K$ are respectivelly the state, input, output and noise matrix to be estimated.

Based on (1) and for simplicity, the class of systems to be considered is linear discrete-time systems with external disturbances of the form.

$$
\left\{\begin{aligned}
x_{k+1} & = & A x_{k}+B u_{k}+K w_{k} \\
y_{k} & = & C x_{k}
\end{aligned}\right.
$$

An advantage of the N4sid method is that it uses a prediction error based on a the Best Fit (BF) displayed percentage related to the output reproduced by the model [24], the formula used in this regard is:

$$
\text { Bestfit }=\left(1-\frac{|y-\hat{y}|}{y-\bar{y}}\right) \times 100
$$

where $y, \hat{y}$ and $\bar{y}$ are respectivelly the measured, the predicted model and the mean of the output $y$.

The proposed control strategy has been suggested for a greenhouse system case of study, where the main task involves internal temperature regulation using heating and ventilation. To illustrate the dynamical behavior of our system depicted in Figure 1, and for the control strategy purposes mentioned above, the discrete time state space model is further used, where the behaviour of the temperature under the greenhouse process is described by the two state-space formulations as detailled in the next subsections.

\subsubsection{Internal temperature responses to actuators}

The main goal in this section is to use the collected data in order to have linear models that will be used as basics for the mathematical identification as follows. 
- Temperature response to fan

In the present part, we describe the evolution of the internal temperature by exciting the system with a step input of 1.6 Volts that was sent to the fan in order to decrease the air temperature under greenhouse until reaching a steady state. Experimental collected data and the N4sid algorithm under Matlab are engaged to develop the corresponding discrete time state space model matrix for 5 seconds as sample time. The evolution of the measured and simulated inside temperature using the N4sid algorithm is shown in Figure 2:

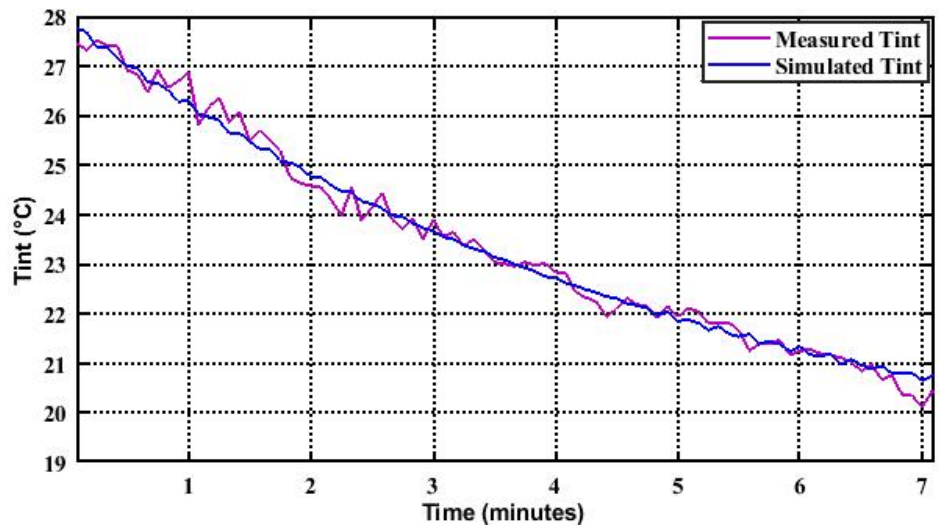

Figure 2. Comparison of simulated and experimental tint step response to a fan

Here the inside temperature reaches its $20.6^{\circ} \mathrm{C}$, where the initial value is $27.9^{\circ} \mathrm{C}$. The model best fit is about $90.34 \%$, hence the state space model identification describes $90.34 \%$ of the behavior of the process output. We can conclude that the simulated and experimental results closely match each other with a good accuracy.

For the controller utilities, the identified model must be converted to a discrete-time system, considering above state space model (2), a discrete linear time invarint system with 3 states, was obtained as follows:

$$
\begin{aligned}
& A_{f}=\left[\begin{array}{llc}
0.9843 & -0.0125 & 0.0100 \\
0.0294 & -0.8090 & 0.6507 \\
0.0032 & -0.6512 & -0.6447
\end{array}\right] \\
& B_{f}=\left[\begin{array}{lll}
-0.0127 & -3.3205 & 4.2182
\end{array}\right]^{T} \\
& C_{f}=\left[\begin{array}{lll}
23.2656 & 1.0200 & -0.1778
\end{array}\right] \\
& D_{f}=0 \\
& K_{f}=\left[\begin{array}{lll}
-0.0152 & -0.0580 & 0.0437
\end{array}\right]^{T}
\end{aligned}
$$

Under the initial state:

$x_{f 0}=\left[\begin{array}{lll}-1.2128 & -0.3375 & -1.4972\end{array}\right]^{T}$

And the open-loop eigen values:

$\sigma\left(A_{f}\right)=\{0.9840,-0.7267 \pm 0.6457 i\}$ 
The index 'f' sticks to above matrix refers to the fan as input actuator engaged in the system state space identification.

- Internal temperature response to heater

The same as in the fan case, the evolution of indoor temperature was also described by exciting the system with a step input of 2.6 Volts that was sent to the heater, hence the air temperature under greenhouse was increased reaching by that a steady state for the same sample time, which is 5 seconds. In this case, the evolution of the measured and simulated inside temperature using the N4sid algorithm is shown in Figure 3:

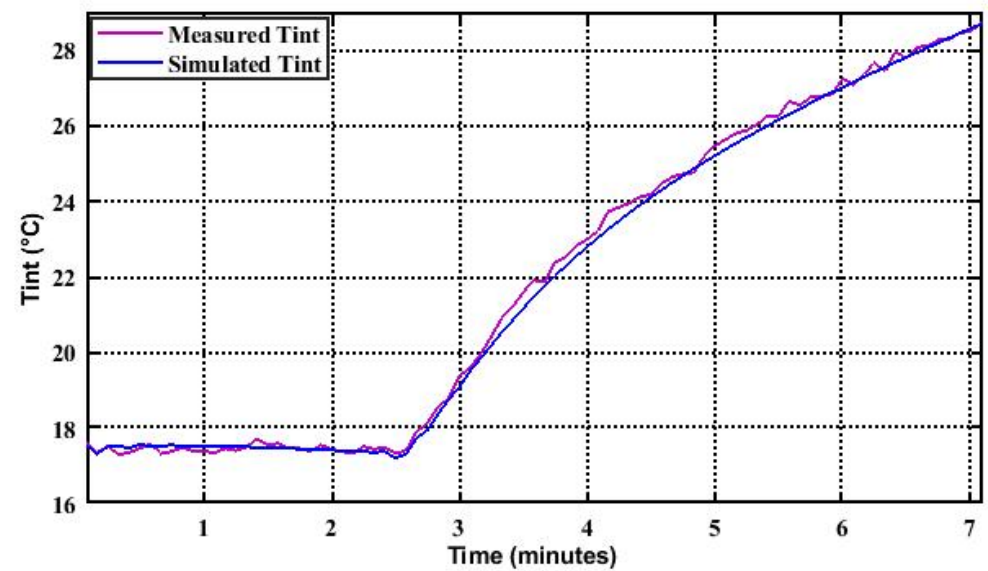

Figure 3. Comparison between simulated and experimental tint step response to a heater

As shown, the inside temperature reaches its $28.5^{\circ} \mathrm{C}$, where the initial value is $17.6^{\circ} \mathrm{C}$. The model best fit this time is $95.5 \%$.

The identified discrete-time system model, with 5 states, was presented as follows:

$$
\begin{aligned}
A_{h} & =\left[\begin{array}{ccccc}
1.0061 & -0.0053 & 0.0034 & 0.0007 & 0.0007 \\
0.0176 & 0.9845 & 0.1607 & -0.0906 & -0.0168 \\
0.0003 & -0.1011 & 0.4634 & -0.8349 & -0.1410 \\
0.0273 & 0.0027 & -0.0009 & -0.2943 & 0.6977 \\
-0.0601 & 0.0194 & -0.2084 & -0.3336 & -0.7432
\end{array}\right] \\
B_{h} & =\left[\begin{array}{llllll}
0.0000 & 0.0263 & 0.0247 & 0.1198 & 0.0384
\end{array}\right]^{T} \\
C_{h} & =\left[\begin{array}{llllll}
184.9508 & 0.8560 & -0.4289 & -0.2221 & 0.2199
\end{array}\right] \\
D_{h} & =0 \\
K_{h} & =\left[\begin{array}{lllll}
0.0036 & -0.0933 & 0.1762 & 0.1326 & 0.0519
\end{array}\right]^{T}
\end{aligned}
$$

Under the initial state:

$$
x_{h 0}=\left[\begin{array}{lllll}
0.0944 & -0.1066 & -0.5345 & 0.2705 & 0.2285
\end{array}\right]^{T}
$$

And the open-loop eigen values: 
$\sigma\left(A_{h}\right)=\{0.9406,0.6172,0.9936,-0.5727 \pm 0.5166 i\}$

The index 'h' refers this time to the heater as input actuator used in the system state space identification as well.

Both identified state space models, indicate that the system is stable, controllable and observable.

\subsection{Controller design}

\subsubsection{MPC and optimization problem brief insight}

In general, MPC controller is a strategy based on an iterative, finite horizon (constrained) optimization of a plant model [25]. An actual or estimated State $x_{k}$ is obtained at each discrete sampling time (k) with the sampled plant model and a cost function is calculated to obtain the performances of the controller in the future based on the current plant state $x_{k}$ and a serie of future inputs $u_{k}$.

The cost function is primordial in predictive controller, that allows us to calculate the best series of control inputs $u_{k}$, which results in a minimal cost in order to keep the output as close as possible to the reference. In control field, having a cost that describes how good our control will be in the future: starting from the next step up to the end of the horizon, is the most important task to take into account. For this aim, a function of the form (4), can be expressed:

$$
J=f\left(x_{k}, u_{k}\right)
$$

Where $x_{k}$ is current state and $u_{k}$ is the control input.

More precisely, the cost function regarding the argument $u_{k}$ has to be minimized in order to get an optimal inputs sequence $u_{k}^{*}$, which can be written as follows:

$$
u_{k}^{*}=\underset{u}{\arg \min } J\left(x_{k}, u_{k}\right)
$$

Which defines an optimal control problem.

The quadratic programming (QP) notion, results in Linear quadratic control, which is related to algorithms based on optimal control as clarified above. The integration of the cost function (5), is chosen to be quadratically dependent on the control input and the state or output response.

In this sense, linear quadratic regulators (LQR) are a special case of the generic linear quadratic control problem, where a gain matrix $\mathrm{K}$ that minimizes an optimization proplem cost function of the form (6), is calculated.

$$
\underset{u}{\operatorname{minimize}} \quad J=\sum_{k=1}^{N} x_{k}^{\prime} Q x_{k}+u_{k}^{\prime} R u_{k}
$$

where $N$ is the prediction horizon, $Q$ and $R$ represent positive-semi definite penality matrices respectively. For more details about (LQR), the reader can refer to [26] and reference therein.

- Quadratic programming parameters

The linear MPC optimization results in quadratic programming (QP) problem. Hence, various are the methods used in this regard such as active-set methods and interior-point methods to solve the problem. Once an optimal solution, i.e., a control input sequence along prediction horizon is numerically obtained, we only use the first element of the sequence as an actual control input.

In addition to MPC parameters, the performance of the predictive controller depends more and more on two other important parameters to be set. These latests are the penalization matrices $Q$ and $R$. Concidering (6), it is remarquabale that both ; the state penalization matrix $Q$, the input penalization matrix $R$ contribution; will affect the desired cost function.

- MPC and constraints contribution

The real meaning of a CMPC lies in computing optimal control actions for systems with constraints contribution [27]. The constraints notion regarding MPC cotroller, could simply be defined as a set of limits on the systems input variables, output or possibly states, which is presented as follows: 


$$
\begin{gathered}
\underline{u} \leq u_{k} \leq \bar{u} \\
\underline{x} \leq x_{k} \leq \bar{x}
\end{gathered}
$$

One can be aware that these constraints have to be reexpressed and evaluated by the mean of quadratic programming (QP) algorithms and suitable solvers. The MPC controller logic and algorighm will remain the same in the presence of constraints, the only thing to be changeable is the method of the optimization. The advantage in this case is that procedure has to be performed at every sampling instant, hence, inputs are computed in a way that they are optimal as possible and guaranteeing closed-loop stability notion. For this aim, the cost function optimization task (6) is rewritten as follows:

$$
\begin{array}{cc}
\underset{u_{k}}{\operatorname{minimize}} \quad J=\sum_{k=1}^{N} x_{k}^{\prime} Q x_{k}+u_{k}^{\prime} R u_{k} \\
\text { subject to } & u_{\min } \leq u_{k} \leq u_{\max }
\end{array}
$$

The suffix "min" and "max" are the lower and upper constraint input bounds.

\subsubsection{The adopted controller}

In the present section, the CDMPC formulation for greenhouse temperature control is presented as a quadratic programming $(\mathrm{QP})$ problem that is solved at each sample time. The conceptual model of the control strategy is depicted in Figure 4.

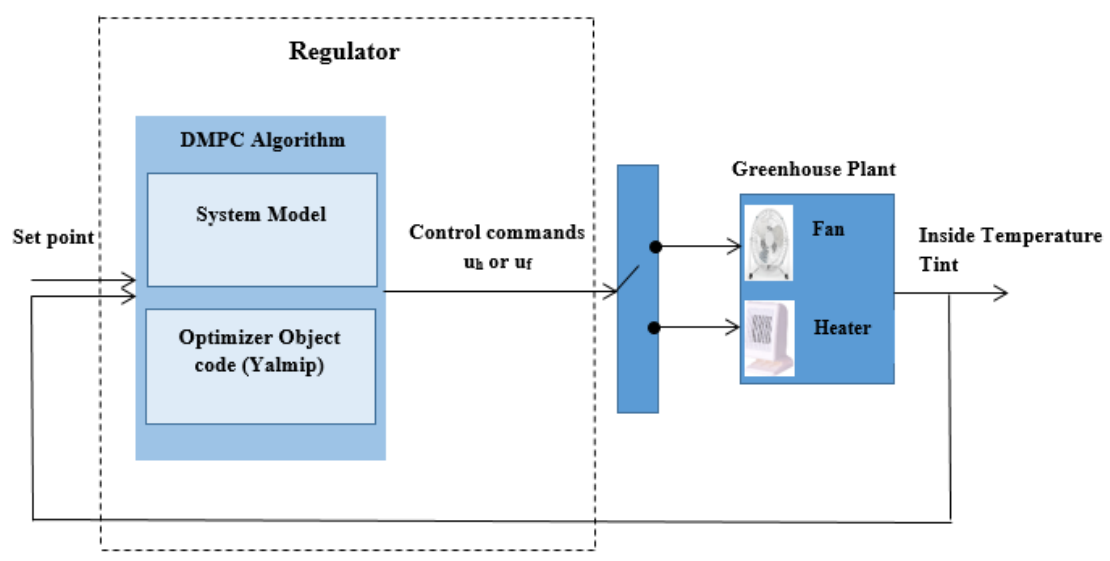

Figure 4. General conceptual model of the proposed control strategy

The constraint on the control notion regarding the system dynamics is brought into the cost function for MPC formulations. Since, the considered system dynamics are linear, the temperature control algorithm would typically involve a linear program (LP) engaging a linear cost function that will be solved using (QP) approach. For this purpose, the cost function aims to penilize any error deviation regarding the inside temperature; which represents the systeme output; and the control input is penelized as well trying to have the optimal control sequence. Regarding this, the main required key elements to take into consideration for an efficient MPC algorithm design are the model and the optimizer. Figure 5 gives an insight of the proposed regulation and optimization procedure.

- The model is one of the most important componements of an MPC algorithm, since it is used to predict the systems bahavior when applying a sequence of control commands, in this end, it has to be as specific as possible for MPC aims. It is worth mentioning that for model based predictive control, both the prediction and the control horizons $\mathrm{Np}$ and $\mathrm{Nc}$, that represent respectivelly the number of predicted future time intervals and the number of control moves for the time interval, are important and have to be 
carrefully adjusted. Those latests contribute directelly in increasing or decreasing both the optimization running and optimization time.

- The optimizer in its part, plays a prominent role in the control approach. Due to Yalmip toolbox used in this framework, the adopted optimizer generates the control activities in the fastest possible way and solves the quadratic programming problem in order to return the optimal solution respecting the constraints on the control notion. In fact, one of the benefits of such a toolbox is that it automatically detects the category of the problem to be solved and optimized in order to select the appropriate solver if available, if it is not the case, the problem is converted to a low level model and then treated and solved.

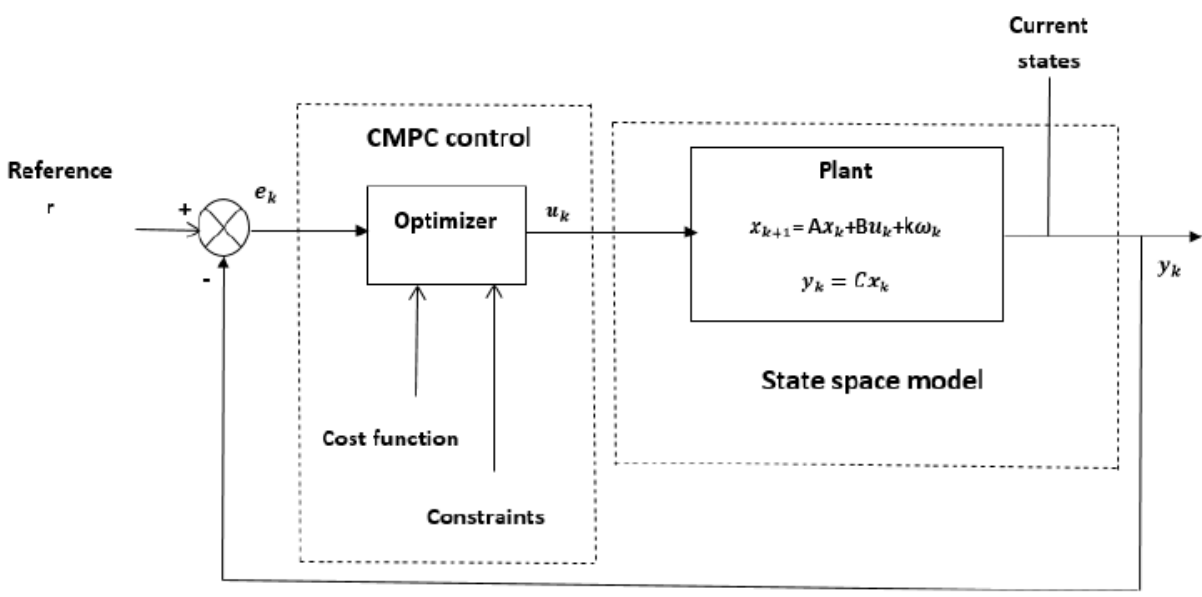

Figure 5. Block diagram of the adopted regulator

In our case of study, single input single output system was taken into account, for this kind of systems, state penality matrix $Q$ may be chosen regarding the states contribution into the cost function, we are aimed to keep the output at a predetermined level, hence the choice of the state penalization matrix was set to provide a kind of states recalculation into outputs $y_{k}$, knowing that $y_{k}=C x_{k}$. For the input penality $R$, it is chosen to be small around $R \approx n^{(-2)}, n \in N$, and depending on the system input contribution in the cost function, one can fix it to lower or higher values. To recall, the constrained optimization problem used in this framework is based on obtaining the control inputs $u_{f}$ and $u_{h}$, i.e., Fan and heater, where a cost function was selected to be quadratically dependent on the systems error $e_{k}$ and the control input $u_{k}$, under the system dynamics and control constraints. The adopted cost function is presented as follows:

$$
\begin{array}{cc}
\underset{u_{k}}{\operatorname{minimize}} \quad J=\sum_{k=1}^{N} e_{k}^{\prime} Q e_{k}+u_{k}^{\prime} R u_{k} \\
\text { subject to } & u_{\min } \leq u_{k} \leq u_{\max }
\end{array}
$$

Where $e_{k}=r-C x_{k}$ and $r$ is the reference value. Here, MPC strategy is implemented in a repeated way such that, at each sampling instant $k$, current states $x_{k}$ are presents first, then, a sequence of future optimal control predictions is calculated and its first element is extracted and applied back to the plant, hence, for each system model, two MATLAB scripts and a simulation model under Simulink were suggested more specifically:

- Firsly, a MATLAB function file code is created, in which the inputs are defined as: the state and the reference, and a scalar control signal is returned as an output.

- Secondely a Simulink model that includes both the linear state-space model and an the interpreted MATLAB function that holds the MPC controller code is set.

- Thirdly, a setup file regarding the state space model data, is also created.

MATLAB 2018b with Yalmip toolbox of the version '20200116' were used to show the simulation developement as presented in section 3. The adopted CDMPC Algorithm using yalmip optimization toolbox for temperature control, can be summarized as follows: 


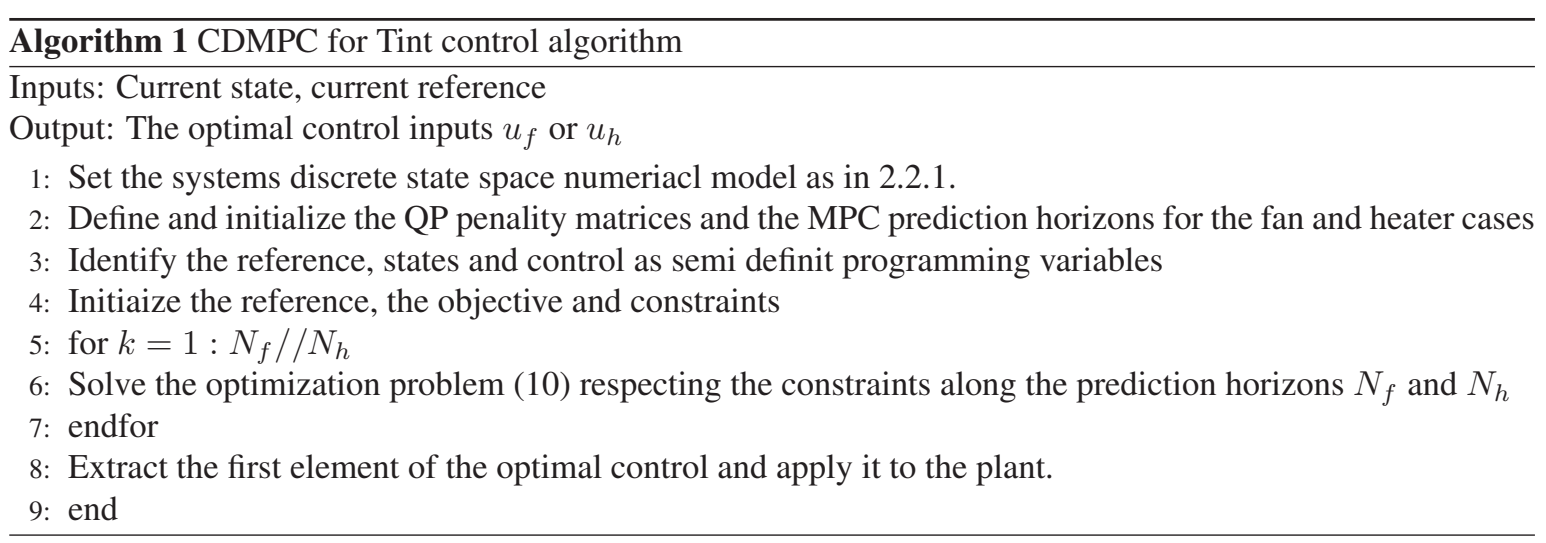

\section{SIMULATION RESULTS AND DISCUSSION}

Numerical simulation was carried out in order to evaluate the CDMPC performances. In this regard, MATLAB, Simulink and model predictive control algorithm using YALMIP Toolbox were used in this frame. The optimization problem is solved by above QP algorithm using QUADPROG as a solver. Interpreted Matlab function was used for the controller, and the plant models for simulation purposes under Simulink was engaged as well.

To recall, the objective of the desired control strategy is to maintain the output $y_{k}$, i.e., inside temperature Tint, as close as possible to the reference value, and try not to exceed posed boundries $15^{\circ} \mathrm{C} \leq$ Tint $\leq$ $25^{\circ} \mathrm{C}$, besides that and for futur real time experiments perspectives, deviding the work in two simulation tasks, i. e., two systems identifications regarding cooling and heating was based essentially on how our system works in real life, i. e., according to the sign of the difference between the setpoint and the measured inner temperature. In order to evaluate the proposed control approach; for both scenarios, i.e., for the fan and heater; the input is constrained to evolve between $0 \leq u_{f} \leq 4.1$ as voltage applied to the fan and $0 \leq u_{h} \leq 5$ as voltage applied to the heater. After some trials, the penality weight factors were chosen scalars as $Q_{f}=100$ and $R_{f}=0.01$ for the first system and $Q_{h}=200$ and $R_{h}=0.2$ for the second one, the prediction horizons were set to $N_{f}=30$ and $N_{h}=40$. For simulation tests, $T_{s}=5 \mathrm{sec}$ was setelled as sample time. Figure 6 describes the evolution of External temperature during 7 minutes, this evolution shows that the external temperature varies between a temperature range $15^{\circ} \mathrm{C}-17.5^{\circ} \mathrm{C}$.

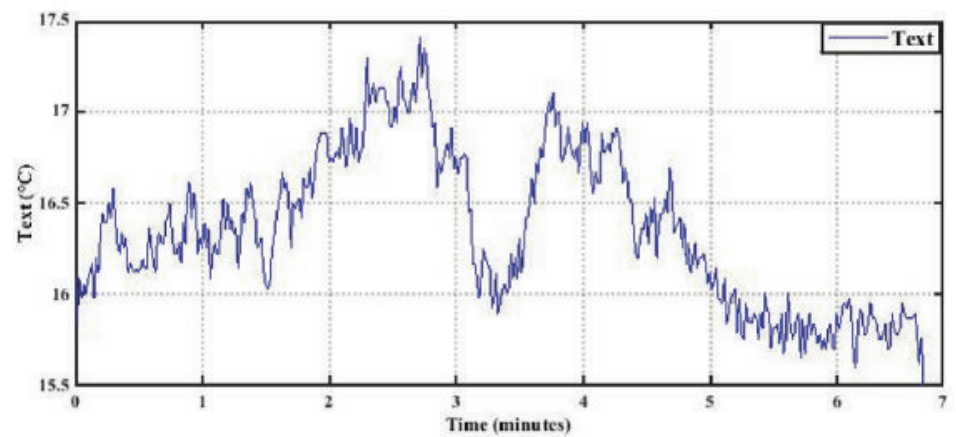

Figure 6. Measured greenhouse external temperature

In one hand, and as presented in Figure 7, it is remarquable that the fans behavior as first actuator, tends to meet the input constraints and more precisely does not exceed 1.5 Volts. Besides, various stopping moments are clearly observed, which contributes to power and energy saving, actuator durability and voltage signal limitation as well. In another hand, Figure 8 shows the the control task peformances, which is eventually noticed in the internal temperature setpoint tracking, respecting the desired temprature limits. We can notice that the temperature decreases from $29^{\circ} \mathrm{C}$ to attend the setpoint variations.

In the same way, Figures 9 and 10 show respectively, the second actuator, i.e., the heater, behavior under constraints and the inside temperature response to the heater input control. As it is visible, the heater 
behaves normally respecting the constraints notion and attempts his maximum/minimum voltage power without exceeding the upper and lower bounds constraints limits. In Figure 9 the control mission was obtained, and the temperature evolves and reachs its $22.3^{\circ} \mathrm{C}$ as first value and then tracks smoothly the setpoint.

It is interesting to note that, the present control strategy regarding the constraints on the control has been evaluated despites some damping comportemnt regarding the setpoint tracking task. In general, one might resume that simulation results using a new optimization toolbox as Yalmip, were succesfully guaranteed. As futur work, various and new are the ideas that has been emerged while working on this article. The first one of them, is the proposed control strategy real time implementation. While the improvement and enhancement of this control method will be taken into account as well. Hoping that these initiatives can lead us to new and interesting results and yields.

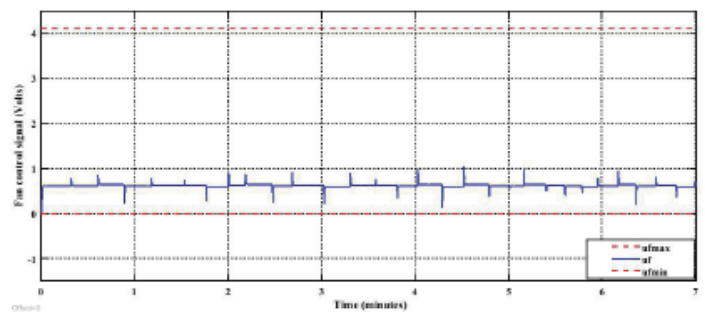

Figure 7. Evolution of the fan control signal under constraints

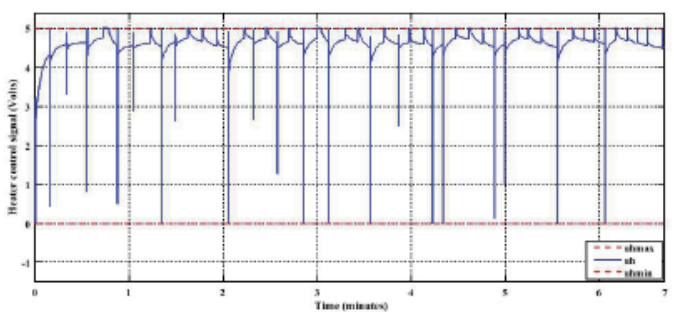

Figure 9. Evolution of the heater control signal under constraints

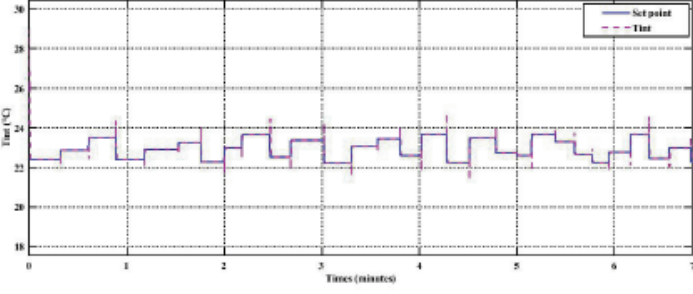

Figure 8. Tint response to the fan as input control "uf"

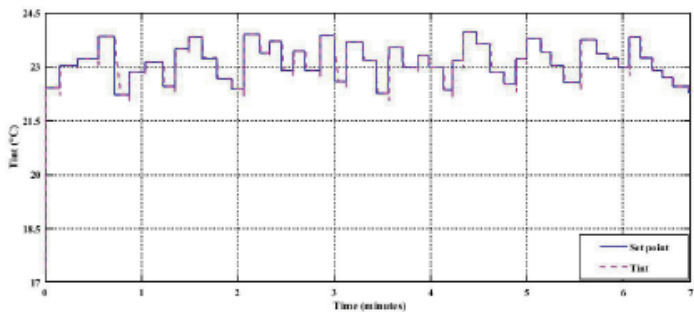

Figure 10. Tint response to the heater input control "uh"

\section{CONCLUSION AND FUTURE PERSPECTIVES}

In this paper, a constrained discrete model predictive control (CDMPC) for discrete time linear SISO system has been considered. The notion of constraints on the control has been treated as well using qadratique programming (QP) optimization algorithm under a novel toolbox as Yalmip. Necessary and sufficient conditions for the synthesis of the elaborated controller that ensure the desired reference signal tracking and control of inside greenhouse temperature; respecting the constraints of the control inputs condition; have been succesfully accomplished and prooved with the above numerical simulations.

We have shown that the control and reference tracking problems are solved for inside temperature of our greenhouse system. The application of these approachs and algorithm can be engaged for other climatic parameter control such as humidity and for multi-input multi-output (MIMO) system as another case of study, in addition to real time implementation, which is one of our perspectives.

\section{REFERENCES}

[1] S. Hemming et al., "Remote control of greenhouse vegetable production with artificial intelligencegreenhouse climate, irrigation, and crop production," Sensors, vol. 19, no 8, pp. 1807, 2019.

[2] H. Mao, C. Jin and Y. Chen., "Research progress and prospect on control methods of greenhouse environment," Chinese Society for Agricultural Machinery, vol. 19, no 2, pp. 1-13, 2018. 
[3] M. Guerboaoui et al., "Implementation of direct fuzzy controller in greenhouse based on labview," International journal of electrical and electronics engineering studies, vol. 1, no 1, pp. 1-13, 2013.

[4] M. Jomaa et al., "Greenhouse Modeling, Validation and Climate Control based on Fuzzy Logic," Engineering, Technology and Applied Science Research, vol. 9, no 4, pp. 4405-4410, 2019.

[5] A. Mirakhorli and B. Dong., "Occupancy behavior based model predictive control for building indoor climate-A critical review," Energy and Buildings, vol. 129, pp. 499-513, 2016.

[6] C. Mao et al., "The research of control method of greenhouse based on global variable prediction model," Chemical Engineering Transactions, vol. 51 pp. 277-282, 2016.

[7] M. Taki et al., "Heat transfer and MLP neural network models to predict inside environment variables and energy lost in a semi-solar greenhouse," Energy and Buildings, vol. 110, pp. 314-329, 2016.

[8] H. Oubehar et al., "ANFIS-based climate controller for computerized greenhouse system," Advances in Science, Technology and Engineering Systems, vol. 5, pp. 8-12, 2020.

[9] C. Lijun et al., "Linear quadratic optimal control applied to the greenhouse temperature hierarchal system," IFAC-PapersOnLine, vol. 51, no 17, pp. 712-717, 2018.

[10] M. R Arahal et al., "Cost function optimization for predictive control of a five-phase IM drive," Optimal Control Applications and Methods, vol. 41, no 1, pp. 84-93, 2020.

[11] U. Eren et al., "Model predictive control in aerospace systems: Current state and opportunities," Journal of Guidance, Control, and Dynamics, vol. 40, no 7, pp. 1541-1566, 2017.

[12] G. Serale et al., "Model predictive control (MPC) for enhancing building and HVAC system energy efficiency: Problem formulation, applications and opportunities," Energies, vol. 11, no 3, pp. 631, 2018.

[13] Y. Ding et al., "Model predictive control and its application in agriculture: A review," Computers and Electronics in Agriculture, vol. 151, pp. 104-117, 2018.

[14] A. Hamza and R. Messaoud., "Non-PDC Interval Type-2 Fuzzy Model Predictive Microclimate Control of a Greenhouse," Journal of Control, Automation and Electrical Systems, vol. 31, no 1, pp. 62-72, 2020.

[15] K. Yoshida, M. Inoue, Hatanaka. T, ”Instant MPC for Linear Systems and Dissipativity-Based Stability Analysis," IEEE Control Systems Letters, vol. 3, no 4, pp. 811-816, 2019.

[16] H. El Bahja et al., "A constrained closed loop MPC based on positive invariance concept for a wastewater treatment plant," International Journal of Systems Science, vol. 49, no 10, pp. 2101-2115, 2018.

[17] A. Bonzanini, T. Santos and A. Mesbah., "Tube-based stochastic nonlinear model predictive control: A comparative study on constraint tightening," Proceedings of the 12th IFAC Symposium on Dynamics and Control of Process Systems, including Biosystems (DYCOPS), vol. 52, no 1, pp. 598-603, 2019.

[18] Y. Wang et al., "Economic model predictive control based on a periodicity constraint," Journal of Process Control, vol. 68, pp. 226-239, 2018.

[19] A. Bonfiglio et al., "An MPC-based Approach for Emergency Control ensuring Transient Stability in Power Grids with Steam Plants," IEEE Trans. on Industrial Elect., vol. 66, no 7, pp. 5412-5422, 2019.

[20] W. Hager et al., "Convergence rate for a Gauss collocation method applied to constrained optimal control," SIAM Journal on Control and Optimization, vol. 56, no 2, pp.1386-1411, 2018.

[21] T. Muhlpfordt et al., "Constrained AC Optimal Power Flow: A Polynomial Chaos Approach," IEEE Transactions on Power Systems, vol. 34, no 6, pp. 4806-4816, 2019.

[22] J. Lofberg., "YALMIP: A Toolbox for Modeling and Optimization in MATLAB," IEEE International Symposium on Computer Aided Control Systems Design, 2004.

[23] A. Eddahhak, et al., "Performance evaluation of a developing greenhouse climate control with a computer system," AMSE Journal Modelling C, vol. 68, no. 1, pp. 53-64, 2007.

[24] M. Carrion et al., "Prediction method for nonlinear time series analysis of air temperature data by combining the false nearest neighbors and subspace identification methods," International Conference on Applied Computer and Applied Computational Science, ACACOS'11, pp. 38-43, 2011.

[25] G. Takacs et al., "Efficiency and performance of embedded model predictive control for active vibration attenuation," European Control Conference, 2016. (ECC) IEEE, pp. 1334-1340., 2017.

[26] M. Outanoute et al., "Synthesis of an Optimal Dynamic Regulator Based on Linear Quadratic Gaussian (LQG) for the Control of the Relative Humidity Under Experimental Greenhouse," International Journal of Electrical and Computer Engineering, vol. 6, no 5, pp. 2088-8708, 2016.

[27] D. Mayne., "Model predictive control: Recent developments and future promise," Automatica, vol. 50, no 12 , pp. 2967-2986, 2014. 


\section{BIOGRAPHIES OF AUTHORS}

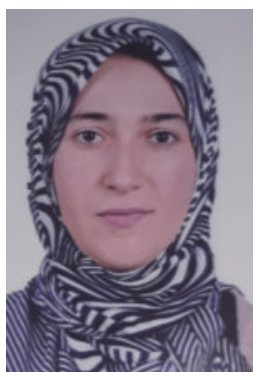

Hafsa Hamidane is a current Ph. D. student and member of Modelling, Materials and Control of Systems team at Laboratory of Electronics Automatics and Biotechnology , Faculty of Sciences, Moulay Ismaill University, Meknes, Morocco. Her research studies are focussed on constrained systems control, pole placement and stability analysis.

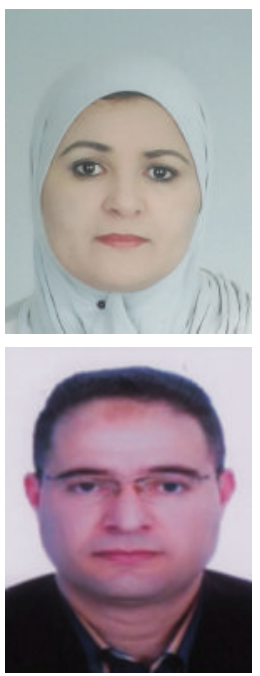

Samira El Faiz is currently a Professor and member of Energy and Sustainable Development Reaserch team in the Department of Electrical Engineering, High School of Technology, Ibn Zohr University, Guelmim. She got her Ph. D. degree from Faculty of Sciences Semlalia, Marrakech in 2004, Her research interest is focussed on Systems Stability and robust pole placement via Linear Matrix Inequalities (LMIs).

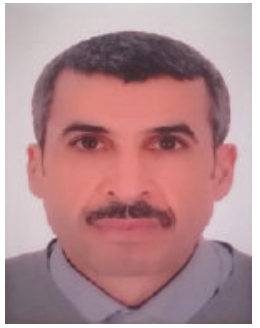

Abdelali Ed-Dahhak is a Professor in the Department of Electrical Engineering, High School of Technology, Moulay Ismail University, Meknes, Morocco. He is a member of Modelling, Materials and Control of Systems team. He got his Ph. D. degree from Faculty of Sciences, Moulay Ismail University, Meknes, in 2009. His current area of research focuses on electronics, development of a system for climate monitoring and managing the drip irrigation in greenhouses.

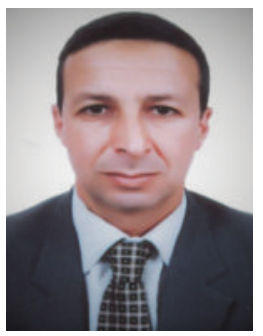

Abdeslam Lachhab is a Professor and the head of Modelling, Materials and Control of Systems team in High School of Technology, Moulay Ismail University, Meknes, Morocco. He received his Ph. D. degree from Faculty of Sciences, Mohammed 5 University, Rabat in 2000. His current research interest includes Systems modelling and automatic control.

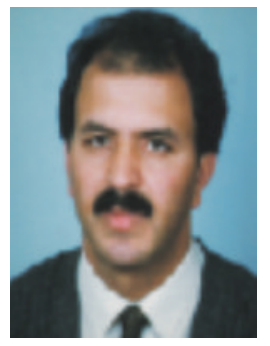

Benachir Bouchikhi received his Ph.D. degree from Law, Economics and Sciences University of Aix, Marseille III, in 1982. Benachir Bouchikhi was awarded as Doctor of Sciences degree in 1988 from the University of Nancy I and got a position of titular professor at the University of Moulay Ismail, Faculty of Sciences, Meknes, Morocco since 1993. He is the director of the Laboratory of Electronics, Automatic and Biotechnology. His current research focuses on the development of electronic nose and electronic tongue devices for food analysis and biomedical applications, besides control of the climate and drip fertirrigation under greenhouse as well. He is author and co-author of over 65 papers, published on international journals. For the last 10 years he has coordinated a dozen national and international projects, in the area of food safety, the control of the climate and drip fertirrigation under greenhouse. He is member of the H2020-MSCA-RISE-2014 project TROPSENSE: "Development of a non-invasive breath test for early diagnosis of tropical diseases. $\mathrm{He}$ is member of the Editorial Board of Journal of Biotechnolgy and Bioengineering. 